\title{
Fruit Volatile Fingerprints Characterized among Four Commercial Cultivars of Thai Durian (Durio zibethinus)
}

\author{
Wattana Aschariyaphotha, ${ }^{1}$ Chalermchai Wongs-Aree, ${ }^{2,3}$ Kitti Bodhipadma, ${ }^{1}$ \\ and Sompoch Noichinda $\mathbb{D}^{1}$ \\ ${ }^{1}$ Division of Agro-Industrial Technology, Faculty of Applied Science, King Mongkut's University of Technology North Bangkok, \\ Bangsue, Bangkok 10800, Thailand \\ ${ }^{2}$ Division of Postharvest Technology, School of Bioresources and Technology, King Mongkut's University of Technology Thonburi, \\ Bangkhuntien, Bangkok 10150, Thailand \\ ${ }^{3}$ Postharvest Technology Innovation Center, Ministry of Higher Education, Science, Research, and Innovation, \\ Bangkok 10400, Thailand
}

Correspondence should be addressed to Sompoch Noichinda; sompoch.n@sci.kmutnb.ac.th

Received 21 May 2021; Revised 18 August 2021; Accepted 24 August 2021; Published 6 September 2021

Academic Editor: Dengyong Liu

Copyright (C) 2021 Wattana Aschariyaphotha et al. This is an open access article distributed under the Creative Commons Attribution License, which permits unrestricted use, distribution, and reproduction in any medium, provided the original work is properly cited.

\begin{abstract}
Ripe durian fruits produce unique volatiles of pungent odor comprising esters, alcohols, ketones, and sulfur-containing compounds. Recently, "Chanthaburi 1" hybrid bred from 2 famous commercial cultivars of "Chanee" and "Monthong" claimed to be less fragrant during ripening, but there was no report. The present study compared the volatile profiles from 3 Thai commercial cultivars of "Kanyao," "Chanee," and "Monthong" compared to "Chanthaburi 1", and the relationships of the cultivars were organized using the volatile fingerprints. Out of 41 volatile compounds detected by SPME/GC-MS in ripe durian flesh, 33 compounds were esters, but only 14 esters were found in "Chanthaburi 1." Ripe flesh of most durian cultivars contains ethyl-2methyl butanoate and ethyl hexanoate as the active volatiles. "Chanthaburi 1" contained fewer components with low odor activity value (OAV) of the volatiles. "Chanee" ripe flesh exhibited the strongest durian smell among the four varieties, whereas "Monthong" exhibited a strong apple-like fruity odor and "Kanyao" was more green fruity. Diethyl disulfide and 3, 5 dimethyl-1, 2, 4-trithiolane contributing pungent smells of garlic or onion were found only in "Chanthaburi 1" and "Monthong." In terms of detected volatiles, "Kanyao" and "Chanee" were highly close when "Monthong" was apart. PCA analysis revealed that "Chanthaburi 1" contained ester compounds ancestrally related to the parents, "Chanee" in the component I and "Monthong" in the component II. These data could be beneficial for managing the status of Thai durians in global markets.
\end{abstract}

\section{Introduction}

In Thailand, durian plant collection was firstly reported for 227 varieties. However, there currently are several cultivars, including "Chanee," "Kanyao," and "Monthong," in the business both in domestic and export markets [1]. "Chanee" comprises a moderate fruit size of $2.5-3 \mathrm{~kg}$. The fruit shape shows swelling in the middle and is blunt at the blossom end with a big and short peduncle. When ripening, fruit is easily peeled, and the yellow flesh is a very soft fine texture, but with the thin flesh and an ample seed, it is famous for domestic markets. "Kanyao" bears a moderate fruit size of $3 \mathrm{~kg}$, showing a round fruit shape and a big and long peduncle. The ripe flesh has smoothly fine texture and is yellow and sweet. "Monthong," the most famous variety, exhibits big fruit of 3-4 kg. Fruit is long, having shoulders at the stem end and protruding at the blossom end. The ripe flesh is dry and thick with a lean seed [2-4]. Ripe fruits of most typical durian varieties release a pungent solid smell, resulting in trouble for foreigners and under public assemblage. Recently, "Chanthaburi 1" $(\mathrm{ICN} \times M$ 5-1-1), bred from "Chanee" and "Monthong," was officially approved and 
registered as a new variety by the Department of Agriculture, Thailand, on 9 October 2006. The fruit is an early-season production with a harvesting time of 99-105 days after pollination. The average fruit weight is $2.5-3 \mathrm{~kg}$, comprising bright yellow flesh and a sweet, delicate texture. The ripe fruit of "Chanthaburi 1" is claimed to have an extra-low smell [5]. Nevertheless, there is no analytical report yet for the volatile characterization of the fruit.

Aroma is a unique character of ripe durian fruit preferred by some but annoying for many people. Furthermore, it is seriously prohibited to take durian fruit/eat during public transportation or in assembly places such as hotels or convention halls. This matter would be a significant obstacle for the marketing of durians. Ethyl esters (fruity esters and general fruit) are the prominent esters in ripe "Monthong" flesh $[6,7]$. Nevertheless, this sweet smell is interrupted by sulfurous smells of sulfur-containing compounds. Ethanethiol, diethyltrisulfide, diethyldisulfide, dimethyl sulfide, 2,3butanedithiol, ethyl 1-methylethyl disulfide, 3-methyl-thiozolidine, methyl ethyl disulfide, and 1-propanethiol are such sulfur-containing compounds found in ripe durian flesh [6-8]. There is no report of the relationship of durian cultivars by the aroma volatile so far. There have been many reports of volatile components of ripe durians in "Monthong" [6-9], few in "Chanee" [6], but there is no report in "Kanyao" and "Chanthaburi 1." Furthermore, from the fruit's visual appearance, "Chanthaburi 1" fruit shape is very similar to the shape of "Kanyao," leading to confusion by visual appearance. Thus, fruit volatile profiles between the cultivars compared as volatile fingerprints were brought in the interest. Here, the present study was to identify odor characteristics of 4 commercial varieties. Volatiles of "Kanyao" and "Chanthaburi 1" were firstly reported, and the volatile relationship of these four varieties was then investigated.

\section{Materials and Methods}

2.1. Plant Materials and Sample Preparation. Mature durian fruits at $90 \%$ maturation from 4 cultivars, "Chanee" (1.9-2.2 kg) at 15 weeks after anthesis (WAA), "Kanyao" at 18 WAA (1.7-2.0 kg), "Monthong" at 19 WAA $(2.2-2.8 \mathrm{~kg})$, and 'Chanthaburi 1 at 14 WAA (1.4-1.8 kg), were harvested from commercial orchards in Chanthaburi Province, eastern Thailand, between April and June 2018. Fruits were incubated at room temperature $\left(25^{\circ} \mathrm{C}, 70-75 \% \mathrm{RH}\right)$ for natural ripening. Fruit showing initial dehiscence at the blossom end (Supplementary Figure 1), referred to as full ripening, was peeled, and the ripe flesh was used for volatile analysis. The visual appearance of the whole fruit and half-dehusked of ripe fruits of the four cultivars is shown in Figure 1.

2.2. Chemicals. The internal standard of volatile analysis was thiophene ( $\geq 99 \%$ purity) (Sigma Chemical Co., USA).

2.3. Volatile Trapping. The ripe aril of each cultivar was finely blended by using a high-speed homogenizer for $2 \mathrm{~min}$. Homogenate at $5 \mathrm{~g}$ was put into a $20 \mathrm{~mL}$ glass vial sealed with a screw cap having a silicone laminated with polytetrafluoroethylene septum. The volatiles in the sample's headspace were trapped by SPME and analyzed by GC-MS modified from [10]. The volatiles in the headspace of the sample in a vial were trapped by solid-phase microextraction (SPME) coated with $65 \mu \mathrm{m}$ of Polydimethylsiloxane/ Divinylbenzene (PDMS/DVB) $(1 \mathrm{~cm}$ length), while heated at $50^{\circ} \mathrm{C}$ for $30 \mathrm{~min}$.

2.4. Analysis of Volatiles in Ripe Durian Flesh. The SPME was injected into a gas chromatogram (GC 6850 series, Agilent Technologies, USA), equipped with an HP-5MS column ( $5 \%$ phenyl-methylsiloane capillary column, $30 \mathrm{~m} \times 0.248 \mathrm{~mm}$ I.D. with $0.25 \mu \mathrm{m}$ thickness) and an Agilent 5913 mass selective detector with the following condition: $200^{\circ} \mathrm{C}$ of the injection port (splitless mode), $50^{\circ} \mathrm{C}$ of the column oven for $1 \mathrm{~min}$ and increased at a rate of $5^{\circ} \mathrm{C} \cdot \mathrm{min}^{-1}$ to $120^{\circ} \mathrm{C}$ and then to $250^{\circ} \mathrm{C}$ at a $10^{\circ} \mathrm{C} \cdot \mathrm{min}^{-1}$ rate, and $250^{\circ} \mathrm{C}$ of the detector. Helium was the carrier gas set to $2 \mathrm{~mL} \cdot \mathrm{min}^{-1}$ at $15.9 \mathrm{psi}$.

Thiophene at $10 \mu \mathrm{L} \cdot \mathrm{L}^{-1}$ was used as the internal standard. The spectra of the volatile profile were analyzed in the electron impact (EI) mode with an electron energy of $70 \mathrm{eV}$; a mass range of $\mathrm{m} / \mathrm{z} 45-450$; a scan rate of $0.25 \mathrm{~s} / \mathrm{scan}$; and an electron multiplier (EM) voltage of $3000 \mathrm{~V}$. Spectra of the volatile profile were compared to a mass spectral database from the NIST V.14 Llibrary values (Palisade Corp., Newfield, NY, USA). There were 3 replications for each analysis.

2.5. Calculation of Volatile Compounds. Each volatile compound of the clear peak from the GC-MS chromatogram was analyzed for the content compared to thiophene as the internal standard. Volatile content in $\mathrm{ng}$ thiophene per $\mathrm{g}$ fresh weight was estimated by the peak area of volatiles divided by the peak area of internal standard (thiophene) and $10 \mu \mathrm{L}$ internal standard solution $\left(0.5 \mathrm{~g} \cdot \mathrm{L}^{-1}\right.$ thiophene $)$ to $5 \mathrm{~g}$ durian homogenate prior to taking SPME [11].

Volatile content $\left(\right.$ ng thiophene $\left.\mathrm{g}^{-1} \mathrm{FW}\right)=\frac{\text { peak area of volatile/peak area of internal standard }}{\text { g durian aril homogenate }}$. 


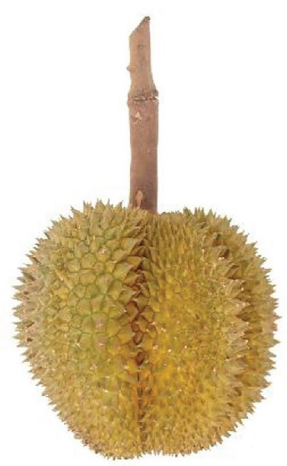
$10 \mathrm{~cm}$

(a)
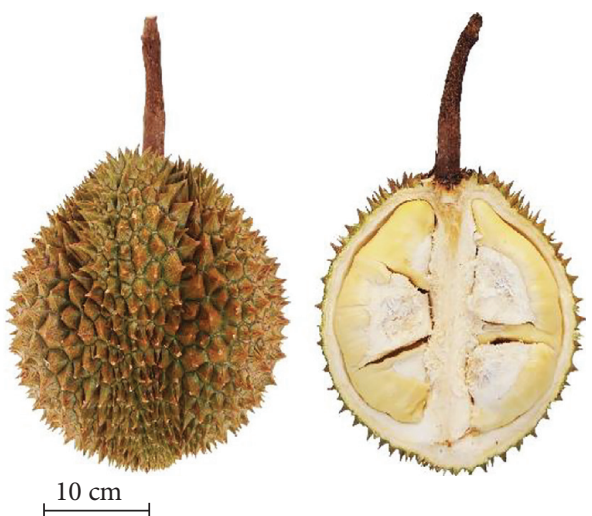

(c)

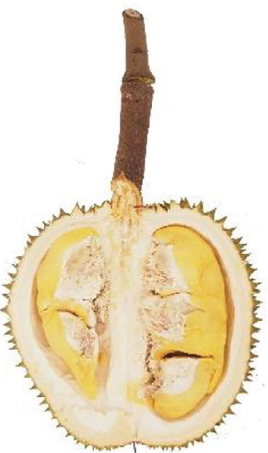

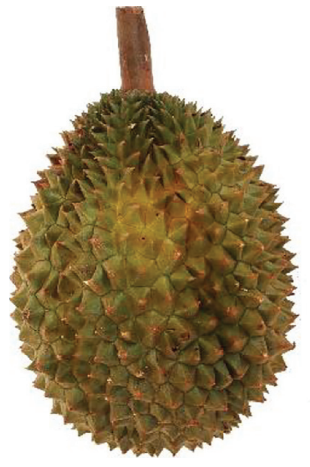

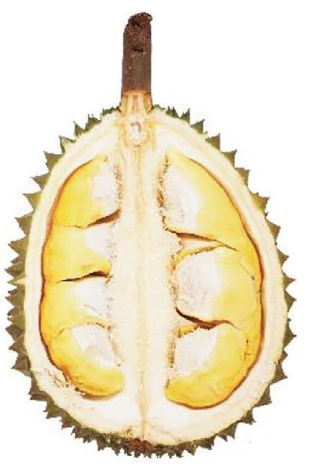

$10 \mathrm{~cm}$

(b)
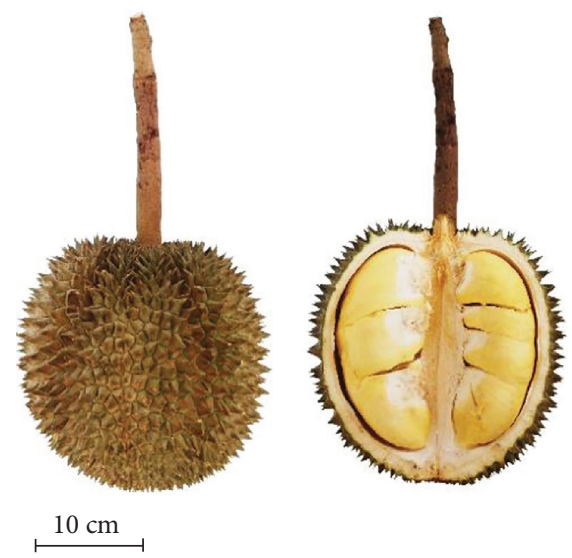

(d)

Figure 1: Appearances of the whole fruit (left) and flesh (right) of (a) “Chanthaburi 1,” (b) "Chanee,” (c) "Monthong," and (d) "Kanyao.”

Odor activity value (OAV) was obtained by dividing the concentration of the compound in a matrix by its odor threshold in that matrix. Thus, it is generally assumed that the odorants with higher OAVs contribute more strongly to the overall aroma. OAV of each volatile compound was calculated using the following formula [12]:

$$
\mathrm{OAV}=\frac{\text { concentration of the volatile content }}{\text { odor theshold value }}
$$

2.6. Statistical Analysis. The volatile relationship of durian cultivars was analyzed using principal component analysis (PCA) by Minitab ${ }^{\circledR}$ program ver.17 (Minitab Ltd., UK). The contents and types of ester volatiles between cultivars were analyzed using multivariations of principal components by Minitab $^{\circledR}$.

\section{Results and Discussion}

3.1. Volatile Profiles in Ripe Durians. From our experience, here was the first report of volatiles contributed in ripe flesh of "Kanyao," and a new hybrid, "Chanthaburi 1" bred from "Chanee" as the female gamete and "Monthong" as the male gamete. There were 41 major volatile compounds detected in
4 cultivars, comprising 33 esters, 2 sulfur-containing compounds, 3 organic acids, 2 phenolics, and 1 aldehyde (Table 1). "Chanthaburi 1" contained major 16 volatiles of 14 esters and 2 sulfur compounds. "Chanee" contained mainly 21 compounds of 17 esters, 3 organic acids, and 1 aldehyde. "Monthong" comprised 23 compounds of 19 esters and 2 sulfur compounds and 2 organic acids. "Kanyao" found 29 volatiles, including 23 esters, 1 sulfur compound, 1 phenolic acid, 3 organic acids, and 1 aldehyde.

Fruit odor is a mixture of many volatile substances, but the main volatile component is the criteria used to determine the odor matter. Nowadays, the odor threshold value of that substance is academically used and can be described, whereas $\mathrm{OAV}$ is calculated from the detected substance. The OAV value greater than 1 is the more important [13]. From OAV, ethyl-2-methylbutanoate (277.3), ethyl nonanoate (225.7), ethyl octanoate (204.9), and ethyl hexanoate (115.0) were the active volatiles of "Chanthaburi 1" ripe flesh, whereas diethyl disulfide was only 4.0 (Table 2). In "Chanee" flesh, 5 ethyl esters, ethyl octanoate (3613.6), ethyl dodecanoate (1126.2), ethyl-2-methylbutanoate (923.2), ethyl hexanoate (318.5), and ethyl propanoate (117.0), were among the major active volatiles (Table 2). In "Monthong" ripe flesh, ethyl octanoate (4173.7), ethyl hexanoate (1808.8), methyl octanoate (843.3), and ethyl-2-methylbutanoate (278.3) were high in the OAV (Table 2), while ethyl octanoate (4241.9), ethyl dodecanoate 
TABLE 1: Volatile compounds released from ripe flesh of 4 Thai durian fruits corresponded to the GC-MS chromatogram profiles.

\begin{tabular}{|c|c|c|c|c|c|c|}
\hline & \multirow{2}{*}{ Compound } & \multirow{2}{*}{ RT } & \multicolumn{4}{|c|}{ Relative content (ng thiophene/g FW) } \\
\hline & & & "Chanthaburi 1" & "Chanee" & "Monthong" & "Kanyao" \\
\hline \multicolumn{7}{|c|}{ Ester } \\
\hline 1 & Methyl-2-methylbutanoate & 0.4766 & nd & nd & 0.71 & nd \\
\hline 2 & Ethyl acetate & 2.1226 & 8.81 & nd & nd & nd \\
\hline 3 & Ethyl propanoate & 2.9628 & nd & 3.39 & nd & 5.70 \\
\hline 4 & Ethyl-2-methylpropanoate & 3.6143 & 1.85 & 5.32 & 2.85 & 9.71 \\
\hline 5 & Ethyl butanoate & 4.3402 & nd & 1.08 & 2.07 & 2.69 \\
\hline 6 & Propyl propanoate & 4.5230 & nd & nd & nd & 2.38 \\
\hline 7 & Ethyl-2-methylbutanoate & 5.3346 & 83.20 & 276.97 & 83.49 & 198.86 \\
\hline 8 & Methyl-2-methyl-2-butenoate & 5.7576 & 4.67 & 1.11 & 1.08 & 1.07 \\
\hline 9 & Methyl hexanoate & 5.7747 & nd & nd & nd & 7.33 \\
\hline 10 & Ethyl-3-methyl-2-butenoate & 6.5005 & 4.74 & 4.98 & 1.51 & nd \\
\hline 11 & Propyl-2-methylbutanoate & 6.5348 & nd & 45.52 & nd & nd \\
\hline 12 & Ethyl-2-methyl-2-butenoate & 7.5865 & 50.87 & 8.30 & nd & 6.61 \\
\hline 13 & Pentyl-2-methylbutanoate & 7.7465 & 26.58 & nd & nd & 63.31 \\
\hline 14 & Methyl-2-hexenoate & 8.3524 & nd & nd & 0.59 & nd \\
\hline 15 & Ethyl hexanoate & 9.2496 & 10.35 & 28.67 & 162.79 & 65.76 \\
\hline 16 & Methyl heptanoate & 9.9754 & nd & 1.93 & nd & nd \\
\hline 17 & Ethyl-2-methylpentanoate & 10.227 & nd & 5.82 & nd & nd \\
\hline 18 & Propyl-2-methyl-(E)-2-butenoate & 10.296 & 16.93 & nd & nd & 3.17 \\
\hline 19 & Ethyl-2-hexenoate & 10.547 & 1.05 & nd & 7.62 & 1.32 \\
\hline 20 & Propyl hexanoate & 11.999 & nd & nd & 18.92 & 10.29 \\
\hline 21 & Ethyl heptanoate & 12.096 & nd & 15.50 & 8.20 & 8.93 \\
\hline 22 & Methyl octanoate & 12.850 & nd & nd & 21.08 & 26.94 \\
\hline 23 & Ethyl-4-octenoate & 14.548 & nd & nd & 3.23 & nd \\
\hline 24 & Ethyl octanoate & 14.931 & 8.20 & 144.54 & 166.95 & 169.68 \\
\hline 25 & Ethyl-2-methyl octanoate & 15.754 & nd & 3.16 & nd & 0.80 \\
\hline 26 & Ethyl-(E)-2-octenoate & 16.302 & 0.85 & nd & 11.35 & 3.10 \\
\hline 27 & 2-Methylbutyl hexanoate & 16.463 & nd & nd & 1.16 & nd \\
\hline 28 & Propyl octanoate & 17.543 & nd & 5.81 & 10.61 & 16.20 \\
\hline 29 & Methyl decanoate & 18.400 & nd & nd & 3.08 & 5.99 \\
\hline 30 & Ethyl decanoate & 20.252 & 2.93 & 4.37 & 24.15 & 27.25 \\
\hline 31 & Methyl dodecanoate & 23.424 & nd & nd & nd & 1.78 \\
\hline 32 & Ethyl dodecanoate & 25.064 & nd & 2.25 & nd & 8.28 \\
\hline \multirow[t]{2}{*}{33} & Ethyl nonanoate & 25.076 & 2.26 & nd & nd & nd \\
\hline & Total & & 223.29 & 558.74 & 531.44 & 647.13 \\
\hline \multicolumn{7}{|c|}{ Sulfur compound } \\
\hline 1 & Diethyl disulfide & 7.0606 & 4.69 & nd & 2.48 & nd \\
\hline \multirow[t]{2}{*}{2} & 3,5-Dimethyl-1,2,4-trithiolane & 13.074 & 5.10 & nd & 1.14 & 1.66 \\
\hline & Total & & 9.79 & 0 & 3.61 & 1.66 \\
\hline \multicolumn{7}{|c|}{ Acid } \\
\hline 1 & Propanoic acid & 2.7227 & nd & 1.02 & nd & 2.91 \\
\hline 2 & Hexanoic acid & 8.8038 & nd & 0.72 & 4.45 & 9.92 \\
\hline \multirow[t]{2}{*}{3} & Octanoic acid & 14.302 & nd & 3.47 & 2.86 & 12.57 \\
\hline & Total & & 0 & 5.21 & 7.31 & 25.40 \\
\hline \multicolumn{7}{|c|}{ Phenolic } \\
\hline \multirow[t]{3}{*}{1} & 2,4-Di-tert-butylphenol & 23.138 & nd & nd & 1.72 & nd \\
\hline & 2,5-bis (1,1-Dimethylethyl phenol) & 23.144 & nd & nd & nd & 1.80 \\
\hline & Total & & 0 & 0 & 1.72 & 1.80 \\
\hline \multirow{3}{*}{1} & & & Aldehyde & & & \\
\hline & trans-2-Methyl-2-butenal & 3.4086 & nd & 4.36 & nd & 9.61 \\
\hline & Total & 3.4086 & 0 & 4.36 & 0 & 9.61 \\
\hline
\end{tabular}

(4138.9), methyl octanoate (1077.4), ethyl hexanoate (730.6), ethyl-2-methylbutanoate (662.9), and ethyl propanoate (196.4) were the active volatiles in "Kanyao" (Table 2). There were some volatile compounds detected only in each cultivar. Ethyl acetate (10.0) and ethyl nonanoate (225.7) were only in "Chanthaburi 1," propyl-2-methylbutanoate (n/a), methyl heptanoate (6.7), and ethyl-2-methyl pentanoate (n/a) were only in "Chanee," methyl-2-methylbutanoate (n/a), methyl-2hexenoate (n/a), ethyl-4-octenoate (n/a), and 2-methylbutyl hexanoate (n/a) were only in "Monthong," and propyl propanoate (8.5), methyl hexanoate $(<1)$, and methyl dodecanoate (683.3) were only in "Kanyao." 
TABLE 2: Odor characteristics of ester, sulfur, acid, phenolic, and aldehyde containing compounds from ripe flesh of 4 Thai durian fruits.

\begin{tabular}{|c|c|c|c|c|c|c|c|c|}
\hline & \multirow[b]{2}{*}{ Compound } & \multirow[b]{2}{*}{ Odor description } & \multirow{2}{*}{$\begin{array}{c}\text { Aroma } \\
\text { threshold } \\
\text { values (ppb) }\end{array}$} & \multicolumn{4}{|c|}{ Odor activity values (OAV) } & \multirow[b]{2}{*}{ References* } \\
\hline & & & & $\begin{array}{l}\text { "Chanthaburi } \\
1 "\end{array}$ & "Chanee" & "Monthong" & “Kanyao" & \\
\hline \multicolumn{9}{|c|}{ Ester } \\
\hline 1 & $\begin{array}{l}\text { Methyl-2- } \\
\text { methylbutanoate }\end{array}$ & $\begin{array}{c}\text { Sweet, fruity, apple-like } \\
\text { odor }\end{array}$ & $\mathrm{n} / \mathrm{a}$ & - & - & $\mathrm{n} / \mathrm{a}$ & - & - \\
\hline 2 & Ethyl acetate & $\begin{array}{l}\text { Fruity, sweet, grape- and } \\
\text { rum-like odor }\end{array}$ & 0.88 & 10.014 & - & - & - & $\mathrm{D}$ \\
\hline 3 & Ethyl propanoate & $\begin{array}{l}\text { Green, fruity apple-like } \\
\text { odor }\end{array}$ & 0.029 & - & 117.048 & - & 196.40 & M \\
\hline 4 & $\begin{array}{c}\text { Ethyl-2- } \\
\text { methylpropanoate }\end{array}$ & Fruity & 0.1 & 18.50 & 53.163 & 28.47 & 97.05 & $\mathrm{O}$ \\
\hline 5 & Ethyl butanoate & Fruity, pineapple & 0.2 & - & 5.38 & 10.35 & 13.44 & $\mathrm{H}$ \\
\hline 6 & Propyl propanoate & $\begin{array}{l}\text { Sharp, chemical, pungent, } \\
\text { sweet, fruity }\end{array}$ & 0.28 & - & - & - & 8.48 & M \\
\hline 7 & $\begin{array}{l}\text { Ethyl-2- } \\
\text { methylbutanoate }\end{array}$ & Fruity & 0.3 & 277.329 & 276.97 & 278.31 & 662.88 & $\mathrm{~N}$ \\
\hline 8 & $\begin{array}{l}\text { Methyl-2-methyl-2- } \\
\text { butenoate }\end{array}$ & $\begin{array}{c}\text { Caramel note, ethereal } \\
\text { rum }\end{array}$ & 35 (in water) & $<1$ & $<1$ & $<1$ & $<1$ & $\mathrm{R}$ \\
\hline 9 & Methyl hexanoate & Fruity, pineapple, ethereal & 70 & - & - & - & $<1$ & $\mathrm{O}$ \\
\hline 10 & $\begin{array}{l}\text { Ethyl-3-methyl-2- } \\
\text { butenoate }\end{array}$ & $\mathrm{n} / \mathrm{a}$ & $\mathrm{n} / \mathrm{a}$ & $\mathrm{n} / \mathrm{a}$ & $\mathrm{n} / \mathrm{a}$ & $\mathrm{n} / \mathrm{a}$ & - & - \\
\hline 11 & $\begin{array}{l}\text { Propyl-2- } \\
\text { methylbutanoate }\end{array}$ & Winey & $\mathrm{n} / \mathrm{a}$ & - & $\mathrm{n} / \mathrm{a}$ & - & - & - \\
\hline 12 & $\begin{array}{l}\text { Ethyl-2-methyl-2- } \\
\text { butenoate }\end{array}$ & Sweet fruity, green notes & $\mathrm{n} / \mathrm{a}$ & $\mathrm{n} / \mathrm{a}$ & $\mathrm{n} / \mathrm{a}$ & - & $\mathrm{n} / \mathrm{a}$ & - \\
\hline 13 & $\begin{array}{l}\text { Pentyl-2- } \\
\text { methylbutanoate }\end{array}$ & $\mathrm{n} / \mathrm{a}$ & 12 & 2.22 & - & - & 5.28 & A \\
\hline 14 & Methyl-2-hexenoate & $\begin{array}{l}\text { Fruity green banana } \\
\text { honey }\end{array}$ & $\mathrm{n} / \mathrm{a}$ & - & - & $\mathrm{n} / \mathrm{a}$ & - & - \\
\hline 15 & Ethyl hexanoate & $\begin{array}{l}\text { Apple-like, fruity, } \\
\text { aniseed-like, sweet }\end{array}$ & 0.09 & 115.032 & 318.54 & $1,808.81$ & 730.63 & $\mathrm{H}$ \\
\hline 16 & Methyl heptanoate & $\begin{array}{c}\text { Sweet, fruity and green, } \\
\text { with a waxy apple-like } \\
\text { note }\end{array}$ & 0.29 & - & 6.67 & - & - & $\mathrm{B}$ \\
\hline 17 & $\begin{array}{l}\text { Ethyl-2-methyl } \\
\text { pentanoate }\end{array}$ & $\begin{array}{l}\text { Fruity, green, melon and } \\
\text { waxy with a fatty nuance }\end{array}$ & $\mathrm{n} / \mathrm{a}$ & - & $\mathrm{n} / \mathrm{a}$ & - & - & $\mathrm{J}$ \\
\hline 18 & $\begin{array}{l}\text { Propyl-2-methyl-(E)- } \\
\text { 2-butenoate }\end{array}$ & $\mathrm{n} / \mathrm{a}$ & $\mathrm{n} / \mathrm{a}$ & $\mathrm{n} / \mathrm{a}$ & - & - & $\mathrm{n} / \mathrm{a}$ & - \\
\hline 19 & Ethyl-2-hexenoate & $\begin{array}{l}\text { Fruity, green, pulpy } \\
\text { pineapple and apple }\end{array}$ & 0.14 & 7.462 & - & 54.41 & 9.46 & $\mathrm{C}$ \\
\hline 20 & Propyl hexanoate & $\begin{array}{l}\text { Sweet, fruity, juicy, } \\
\text { pineapple, green and } \\
\text { tropical }\end{array}$ & 70 & - & - & $<1$ & $<1$ & $\mathrm{E}$ \\
\hline 21 & Ethyl heptanoate & $\begin{array}{l}\text { Fruity pineapple cognac } \\
\text { rum wine }\end{array}$ & 0.24 & - & 64.60 & 34.16 & 37.22 & $\mathrm{D}$ \\
\hline 22 & Methyl octanoate & $\begin{array}{l}\text { Waxy, green, sweet, } \\
\text { orange, aldehydic, } \\
\text { vegetable, herbal }\end{array}$ & 0.025 & - & - & 843.29 & 26.94 & I \\
\hline 23 & Ethyl-4-octenoate & $\mathrm{n} / \mathrm{a}$ & $\mathrm{n} / \mathrm{a}$ & - & - & $\mathrm{n} / \mathrm{a}$ & - & - \\
\hline 24 & Ethyl octanoate & $\begin{array}{l}\text { Pleasant, fruity, floral } \\
\text { odor, wine apricot note }\end{array}$ & 0.04 & 204.91 & $3,613.61$ & $4,173.69$ & $1,077.42$ & $\mathrm{~K}$ \\
\hline 25 & $\begin{array}{l}\text { Ethyl-2-methyl } \\
\text { octanoate }\end{array}$ & $\mathrm{n} / \mathrm{a}$ & $\mathrm{n} / \mathrm{a}$ & - & $\mathrm{n} / \mathrm{a}$ & - & $\mathrm{n} / \mathrm{a}$ & - \\
\hline 26 & $\begin{array}{l}\text { Ethyl-(E)-2- } \\
\text { octenoate }\end{array}$ & $\begin{array}{l}\text { Fruity, green with a fatty } \\
\text { waxy note }\end{array}$ & $\mathrm{n} / \mathrm{a}$ & $\mathrm{n} / \mathrm{a}$ & - & $\mathrm{n} / \mathrm{a}$ & $\mathrm{n} / \mathrm{a}$ & - \\
\hline 27 & $\begin{array}{l}\text { 2-Methylbutyl } \\
\text { hexanoate }\end{array}$ & Ethereal & $\mathrm{n} / \mathrm{a}$ & - & - & $\mathrm{n} / \mathrm{a}$ & - & - \\
\hline 28 & Propyl octanoate & $\mathrm{n} / \mathrm{a}$ & $\mathrm{n} / \mathrm{a}$ & - & $\mathrm{n} / \mathrm{a}$ & $\mathrm{n} / \mathrm{a}$ & $\mathrm{n} / \mathrm{a}$ & - \\
\hline 29 & Methyl decanoate & Oily, winey, fruity, floral & $\mathrm{n} / \mathrm{a}$ & - & - & $\mathrm{n} / \mathrm{a}$ & $\mathrm{n} / \mathrm{a}$ & - \\
\hline 30 & Ethyl decanoate & $\begin{array}{l}\text { Fruity, grape-, cognac-, } \\
\text { and brandy-like odor }\end{array}$ & 0.53 & 5.53 & 8.25 & 24.15 & 51.42 & $\mathrm{~F}$ \\
\hline
\end{tabular}


TABLE 2: Continued.

\begin{tabular}{|c|c|c|c|c|c|c|c|c|}
\hline \multirow{2}{*}{\multicolumn{2}{|c|}{ Compound }} & \multirow[b]{2}{*}{ Odor description } & \multirow{2}{*}{$\begin{array}{c}\text { Aroma } \\
\text { threshold } \\
\text { values (ppb) }\end{array}$} & \multicolumn{4}{|c|}{ Odor activity values (OAV) } & \multirow[b]{2}{*}{ References" } \\
\hline & & & & $\begin{array}{c}\text { "Chanthaburi } \\
1 \text { " }\end{array}$ & "Chanee" & "Monthong" & "Kanyao" & \\
\hline 31 & Methyl dodecanoate & $\begin{array}{l}\text { Waxy, soapy nutty, } \\
\text { coconut, mushroom }\end{array}$ & 0.0026 & - & - & - & 683.31 & $\mathrm{~B}$ \\
\hline 32 & Ethyl dodecanoate & $\begin{array}{l}\text { Waxy, soapy, rummy, } \\
\text { nutty, floral }\end{array}$ & 0.002 & - & $1,126.15$ & - & $4,138.90$ & $\mathrm{~B}$ \\
\hline 33 & Ethyl nonanoate & $\begin{array}{c}\text { Slightly fatty, oily, fruity, } \\
\text { nutty, reminiscent of } \\
\text { cognac with a rosy fruity } \\
\text { note }\end{array}$ & 0.01 & 225.68 & - & - & - & $\mathrm{L}$ \\
\hline
\end{tabular}

\begin{tabular}{|c|c|c|c|c|c|c|c|c|}
\hline \multicolumn{9}{|c|}{ Sulphur compound } \\
\hline 1 & Diethyl disulfide & Onion, garlic & 2 & 3.97 & - & 1.25 & - & M \\
\hline 2 & $\begin{array}{l}\text { 3,5-Dimethyl-1,2,4- } \\
\text { trithiolane }\end{array}$ & Sulphury, onion, meaty & $\mathrm{n} / \mathrm{a}$ & $\mathrm{n} / \mathrm{a}$ & - & $\mathrm{n} / \mathrm{a}$ & $\mathrm{n} / \mathrm{a}$ & G \\
\hline \multicolumn{9}{|c|}{ Acid } \\
\hline 1 & Propanoic acid & Pungent, acidic, dairy & 1 & - & 1.02 & - & 2.94 & $\mathrm{P}$ \\
\hline 2 & Hexanoic acid & Sour, fatty, sweaty, cheesy & 0.0047 & - & 154.23 & 956.57 & $2,132.40$ & Q \\
\hline 3 & Octanoic acid & $\begin{array}{l}\text { Fatty, waxy, rancid oily, } \\
\text { vegetable cheesy }\end{array}$ & 0.011 & - & 318.60 & 262.70 & $1,154.29$ & Q \\
\hline \multicolumn{9}{|c|}{ Phenolic } \\
\hline 1 & $\begin{array}{l}\text { 2,4-Di-tert- } \\
\text { butylphenol } \\
\text { 2,5-bis }(1,1-\end{array}$ & $\mathrm{n} / \mathrm{a}$ & $\mathrm{n} / \mathrm{a}$ & - & - & $\mathrm{n} / \mathrm{a}$ & - & - \\
\hline 2 & $\begin{array}{l}\text { Dimethylethyl } \\
\text { phenol) }\end{array}$ & & & - & - & - & $\mathrm{n} / \mathrm{a}$ & - \\
\hline \multicolumn{9}{|c|}{ Aldehyde } \\
\hline 1 & $\begin{array}{l}\text { trans-2-Methyl-2- } \\
\text { butenal }\end{array}$ & Strong green fruit & $\mathrm{n} / \mathrm{a}$ & - & $\mathrm{n} / \mathrm{a}$ & - & $\mathrm{n} / \mathrm{a}$ & G \\
\hline
\end{tabular}

Ripe flesh of most durian cultivars contains ethyl-2methylbutanoate (fruity note) and ethyl hexanoate (fruity, apple, green, and tropical fruit odor) as the active volatiles showing high OAV. Both found in all four cultivars and most commercial durians were blended with some highOAV compounds to characterize the flavor of each durian variety. In general, ripe durian flesh exhibits the fruity sweet fragrance of both compounds. "Chanthaburi 1" contained fewer components of volatiles as well as low OAV of the volatiles. This indicates that the flesh of "Chanthaburi 1" conducted very low intensity of odors during ripening. Ethyl heptanoate (fruity, pineapple, banana-like note) was found in every cultivar except "Chanthaburi 1." Ripe flesh of "Chanee" exhibited the strongest durian aroma among 4 varieties: "Chanee" exhibited aroma of ethyl octanoate (fruity, floral odor, wine apricot note), ethyl dodecanoate (waxy, soapy, nutty, rummy), and ethyl propanoate (green fruity, apple-like) characterized as nutty, rummy, and green apple-like, "Monthong" exhibited strong apple-like fruity, aldehydic, waxy fragrance of ethyl octanoate (fruity, floral odor, wine apricot note), ethyl hexanoate (apple-like, fruity), and methyl octanoate (waxy, green, sweet, orange, aldehydic vegetable), and "Kanyao" exhibited more complex waxy, nutty, green apple-like fruity aroma of ethyl octanoate, ethyl dodecanoate (waxy, soapy, nutty, rummy note), methyl octanoate (waxy, green, sweet orange), and ethyl propanoate (green fruity, apple-like).

Diethyl disulfide and 3,5-dimethyl-1,2,4-trithiolane found in low levels in ripe durian pulp are the key compounds in durians. Although sulfur-containing compounds exhibited low OAV, compared to the esters, they exhibit an annoying pungent smell. "Chanthaburi 1" as well as "Monthong" contained sulfur-containing compounds of diethyl disulfide and 3,5-dimethyl-1,2,4-trithiolane, which exhibit a garlic-like, onion-like, pungent smell [32]. In particular, diethyl disulfide in ripe "Monthong" showing an OAV of 1.25 would release the pungent smell of "Monthong" durian as reported by Laohakunjit et al. [8] and Niponsak et al. [9]. Previous studies in Malaysia and Indonesia found that the indigenous varieties exhibited a prominent smell of sulfur-containing compounds when fully ripe showing an unpleasant odor overall [32, 33].

In 4 cultivars of Thai durian fruit, ripe aril sharply produced a series of ethyl esters derived from ethyl alcohol and acyls $\mathrm{CoA}$ of straight carbons ranging from $\mathrm{C}_{4}-\mathrm{C}_{10}$ (Table 1). Ethanol in the aril could be generated from anaerobic respiration under a partial hypoxic condition in aril tissue. Due to very high respiration of durian fruit during ripening, fruit husk behaving like a gas barrier makes low gas permeability to the aril. Under partial hypoxia, anaerobic respiration was induced in the aril, resulting in increased 
ethanol [34-37]. Aliphatic and aromatic alcohols are typically found in Malaysian durians, whereas thiols are produced in Thai durians and alcohols are not typically produced in Indonesian and Filipino durians [37]. On the other hand, with a series of straight acyl CoA reacted with the ethanol, it is supposed that $\beta$-oxidation of fatty acids would be involved in the process of ripe fruits [38] as durian pulps have high contents of fatty acids such as methyl stearate $(35.93 \%)$, methyl palmitate $(32.91 \%)$, methyl palmitoleate $(9.50 \%)$, methyl octadecenoate $(4.86 \%)$, methyl oleate $(4.68 \%)$, methyl myristate $(2.52 \%)$, and methyl linoleate $(2.20 \%)$ [39]. Furthermore, amino acid metabolism plays a crucial role in ester production in durians. For instance, ethyl-2-methylbutanoate, a primary volatile compound, is derived from 2-methylbutanoyl-CoA through isoleucine metabolism [40]. The origination of acyls CoA in the ester production could be separated into two sources from the results. When the acyl CoA of $\mathrm{C}_{4}$ could be derived from amino acids, acyl $\mathrm{CoA}$ above $\mathrm{C}_{6}$ could be from lipid oxidations. Furthermore, alcohol acyltransferase (AAT), which modifies alcohols and acyl CoA to esters, could be essentially involved in the production of esters in most durians. Although ATT has not yet been reported in durian, it was reported to be essential for ester production during ripening in many fruits [41-43]. However, as a result of fewer esters in "Chanthaburi 1," the production of esters is apparently disturbed in the fruit probably by mutant functioning of the AAT or the substrate-enzyme incompatibility.

\subsection{The Relationship of Durian Cultivar Relied on Aroma} Volatiles. All 4 varieties showed that ethyl esters were the major components in the ripe flesh. Ethyl acetate and ethyl nonanoate were found in "Chanthaburi 1" but not in the parent, "Chanee" and "Monthong," whereas, on the other hand, ethyl butanoate and ethyl heptanoate found in the parent were not found in "Chanthaburi 1". Methyl-2-methylbutanoate was detected only in ripe "Monthong" flesh.

Ester compounds as the major volatiles were taken to calculate the relationship between cultivars. The differences in essential substances between durian species may be due to genetics and the environment. Genetic factors influence the formation of precursors, enzymes, and odor generation [44]. The durian of "Chanthaburi 1," a hybrid variety, has overall odor characteristics related to the parent variety, "Chanee," and the father species is "Monthong." Nevertheless, by considering the odor, "Chanthaburi 1" has a mild odor while still unripe, similar to the odor of "Kanyao." Although identifying the essential substances in "Chanthaburi 1" durian exhibited a more minor odor type than the strong aroma varieties, the essential substances (OAV) in the "Chanthaburi 1" exhibited characteristics related to both "Chanee" and "Kanyao." The relative content of the ester was obtained according to the dendrogram (Figure 2) of each essential substance. The volatile contents in "Chanthaburi 1" were related to "Monthong" when considering the ester composition. The ester compounds in "Chanthaburi 1" were correlated well with the "Monthong" variety, consistent with the species characteristics that 'Monthong' was the father. However, the relationship of ester compounds in "Chanee" was close to that in "Kanyao."

Principal component analysis (PCA) using the ester compounds from Table 1 was operated to correlate and classify the essential components of the four durian varieties. Ester compounds were classified in the same component, with an eigenvalue greater than 1 , and the component was equal to 2 (data not shown) with Minitab $\odot 17$ displayed in the score plot and biplot (Figure 3). The main component and the secondary components were associated with the ester compounds of the four durian varieties. When looking at the main components, "Chanthaburi 1" durian was related to "Chanee," and from the secondary, "Chanthaburi 1" was, on the other hand, related to the "Monthong" variety which corresponds to the ester characteristics of the parents. But, "Kanyao" has characteristics that are clearly different from those of "Chanthaburi 1" by both components. In addition, the ester characteristics of "Chanthaburi 1" as shown in Figure 3(c) were ethyl acetate, ethyl nonanoate, and methyl2-methyl-2-butenoate, which exhibit a rum-like, grape, and cognac, as well as caramel note. For "Chanee," it can be seen from Figure 3(c) that the distinctive esters were propyl-2methylbutanoate, ethyl-2-methyl pentanoate, and methyl heptanoate showing winey, apple, pineapple, green, melon and waxy flavors, cognac rum wine, intensely fruity, and orris-like. In "Monthong," the ester characteristics were methyl-2-methylbutanoate, ethyl-2-hexenoate, and methyl2 -hexenoate. The scent characteristics are sweet fruity, apple-like odor, green, pineapple, apple, green, banana, honey. On the other side, "Kanyao" exhibited a distinctive scent of methyl hexanoate, propyl propanoate, and methyl dodecanoate, showing fruity, pineapple, complex fruity odor, apple and banana, waxy soapy, nutty, and coconut mushroom. When considering the OAV value of each durian species, if the OAV is greater than 1 , it can be expected to exhibit a unique aroma. The OAV value of "Chanthaburi 1" was clearly similar to that of "Chanee," the mother variety, and close to that of "Kanyao" (Table 2 and Figure 4). The OAV values showed that "Chanthaburi 1" had the dominant esters, ethyl acetate (10.0) and ethyl nonanoate (225.7), which exhibited fruity, sweet, grape and rum-like, slightly fatty, oily, fruity, scent characteristics of nutty, reminiscent of cognac with a rosy fruity note. Nevertheless, "Kanyao" has outstanding OAV values of ethyl octanoate $(4,241.9)$ and ethyl dodecanoate $(4,138.9)$ at high, which is likely to be another distinctive scent, characterized by long stems showing fruity, fatty, floral odor (wine apricot note), waxy, sweet, musty, pineapple, dairy, sweet, waxy soapy rummy, and nutty floral. The distinctive OAV value is of methyl heptanoate (6.7) because it is found only in "Chanee," showing sweet, fruity, and green, with a waxy apple-like note. The higher levels of OAV were found in "Monthong" and "Kanyao" durians, but less common in "Chanthaburi 1" was ethyl octanoate (204.9), which showed a pleasantly fruity, floral odor (wine apricot note). The OAV values were different from the ester relative content, which was the relative content of the volatile compounds present in each durian species, indicating that "Chanthaburi 1" was 


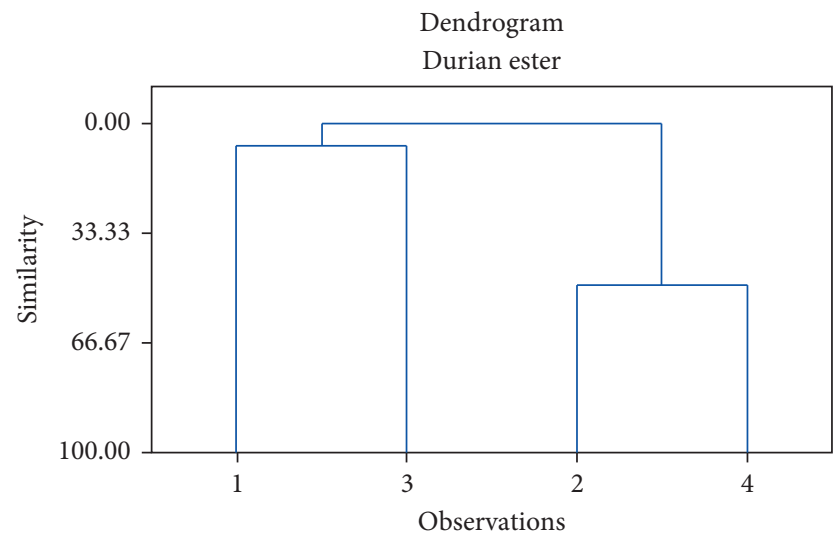

FIGURE 2: Dendrogram of the relationship in the ester compound produced in 4 Thai durian cultivars $(1=$ "Chanthaburi 1 ," $2=$ "Chanee," $3=$ "Monthong," and $4=$ "Kanyao").

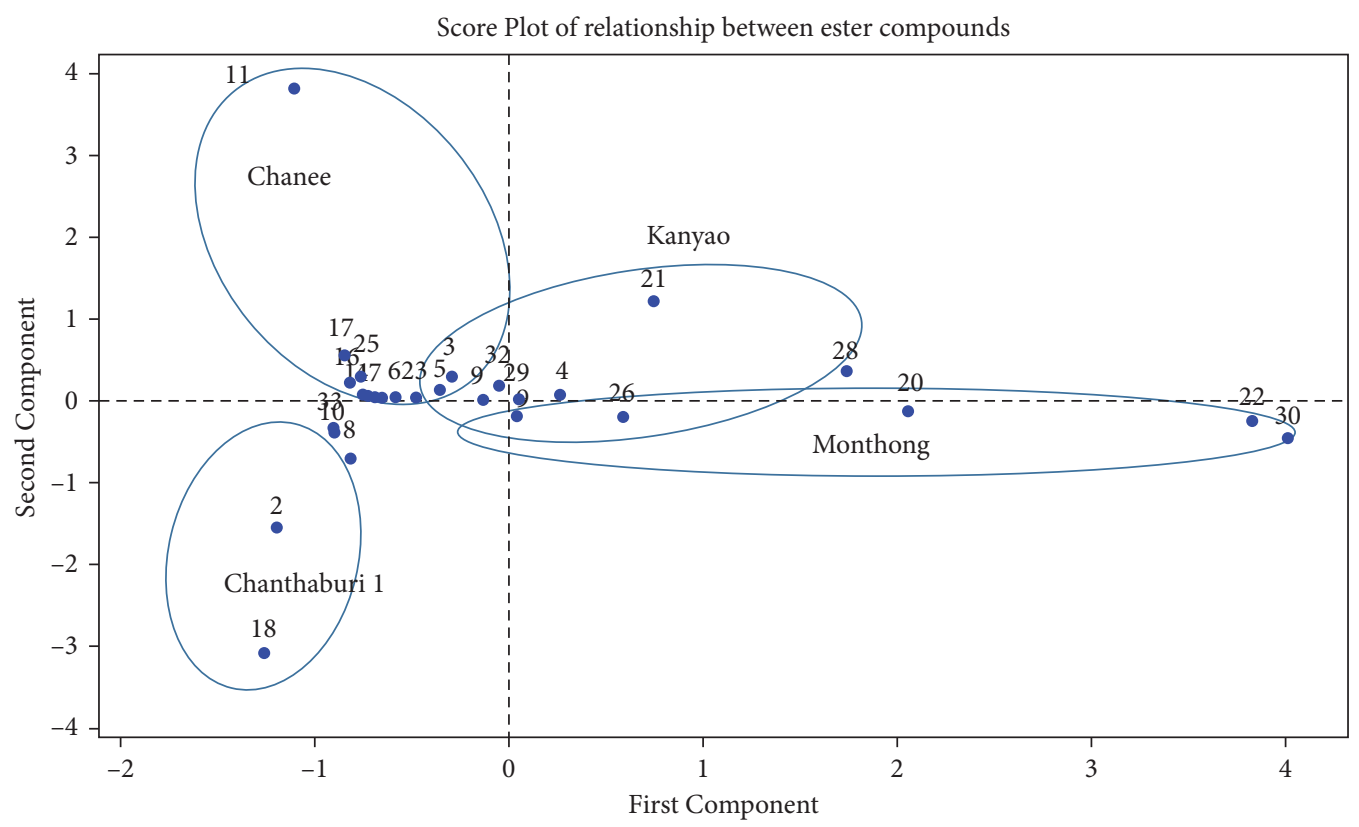

(a)

FIGURE 3: Continued. 


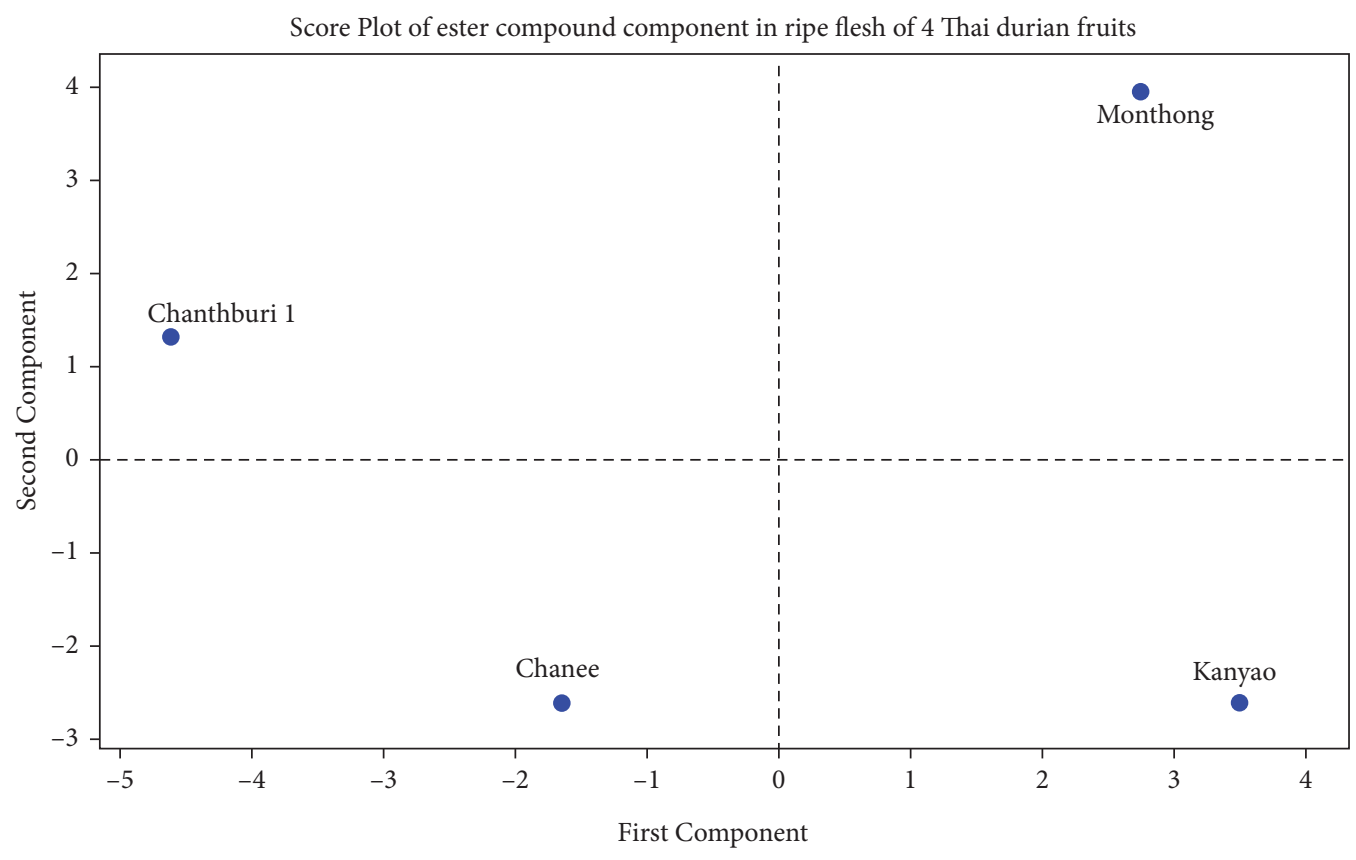

(b)

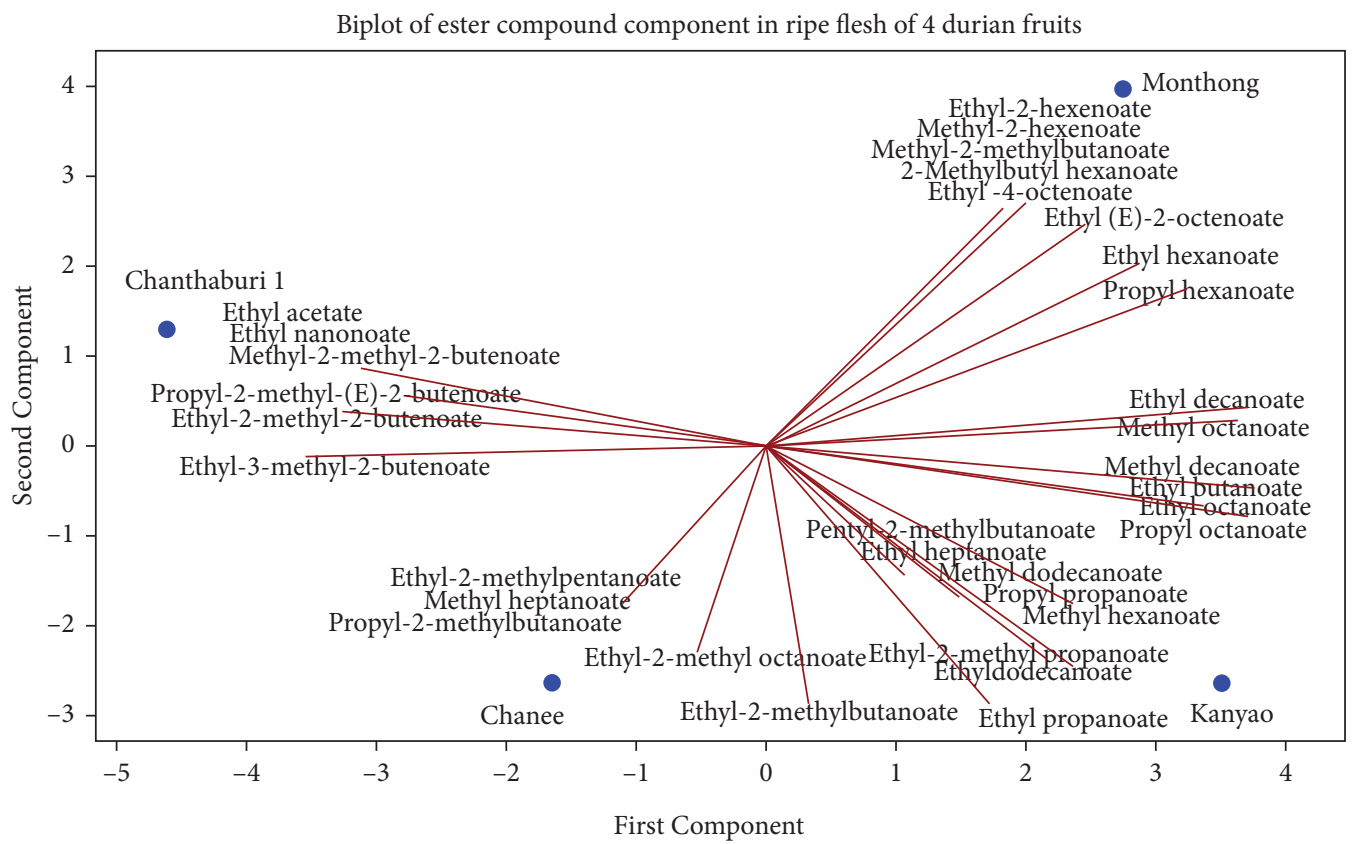

(c)

FIgURE 3: Principal component analysis (PCA) relationship between 4 Thai durian cultivars. (a) Score plot of the relationship between 4 Thai durian cultivars using the ester compounds. (b) Score plot of the ester compound component in ripe flesh of 4 Thai durian cultivars. (c) Biplot of the ester compound component relationship with 4 Thai durian cultivars. 


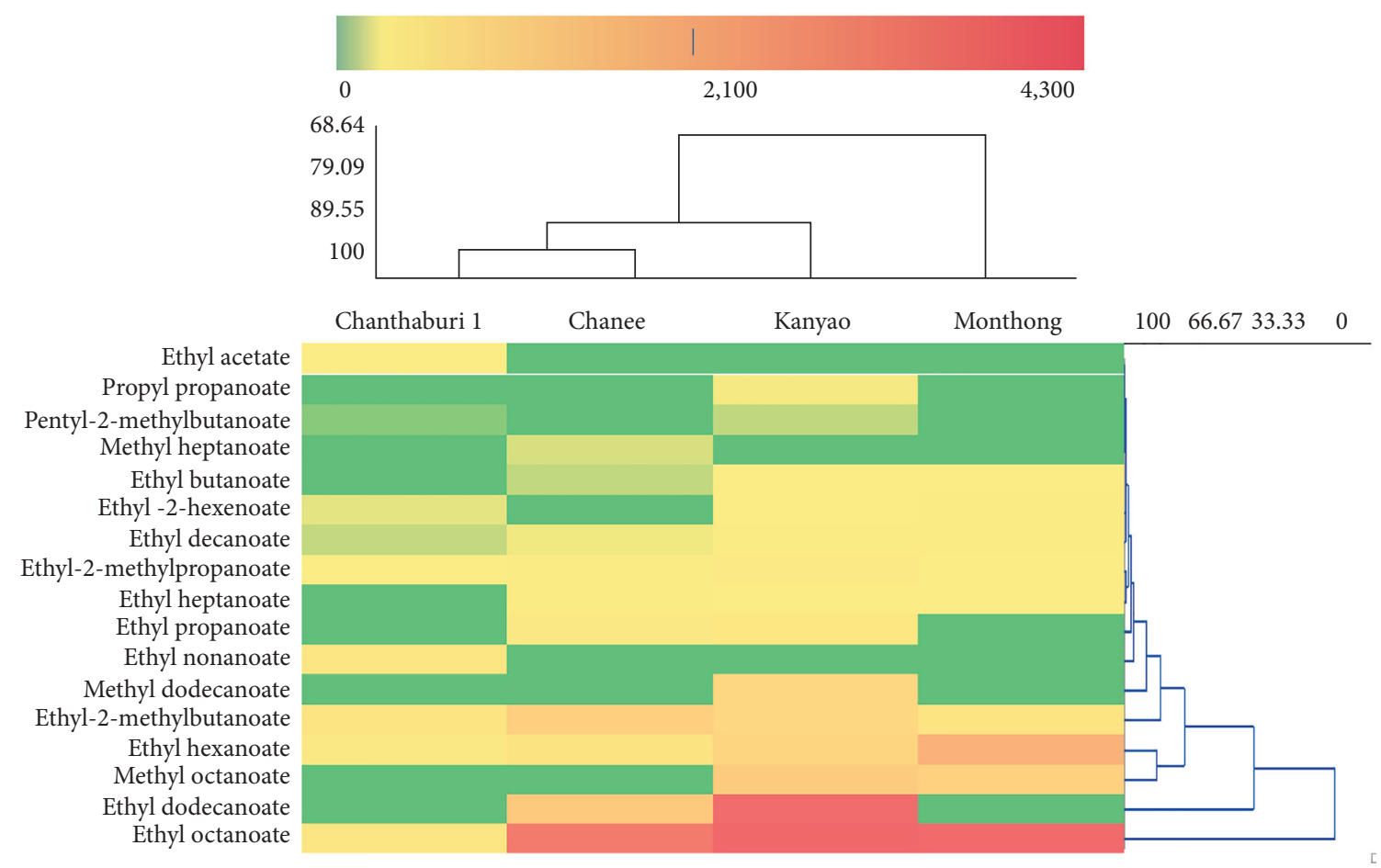

FIGURE 4: Heat map of odor activity value (OAV), which is greater than 1 of each durian species.

consistent with "Monthong" and "Chanee," the father and mother, respectively. Nevertheless, if the OAV value was considered, "Chanthaburi 1" was close to "Kanyao" more than "Monthong" (Figure 4). According to the observation from the odor characteristics, the odor of "Chanthaburi 1" is mild, similar to that of "Kanyao," which the OAV value can explain to some extent. Based on information on the composition of these essential substances, it could greatly benefit the status of Thai durians in terms of the choice of eating fresh fruit and the choices to use ripe durian pulp as an ingredient of food or dessert which requires the durian odor. The study could increase the opportunities of Thai durian transport channels to the world.

\section{Conclusions}

Thirty-three esters and three sulfur-containing compounds were the main volatiles found and affected the flavor character of the ripe pulp of four varieties of Thai durians, "Chanee," "Monthong," "Kanyao," and "Chanthaburi 1." Ethyl esters were the major esters as ethyl-2methylbutanoate and ethyl hexanoate were the crucial essential substances found in all four varieties. The overall aroma character of the durian was a mixture combined of fruity-like apple/pineapple with rum, butter, oily, and waxy odors. Although ripe durians produced few sulfurcontaining volatiles, the compounds exhibit a sulfurous pungent smell. Using the volatile ester profiles, "Chanthaburi 1" correlated with "Chanee," the mother breed, and "Monthong," the father breed. "Kanyao" was different from "Chanthaburi 1." However, with high OAV values concerned, "Chanthaburi 1" was obviously associated with "Chanee," but the odor character was more similar to "Kanyao" than "Monthong."

\section{Data Availability}

The data used to support the findings of this study are included within the supplementary information file.

\section{Conflicts of Interest}

There are no conflicts of interest in this study.

\section{Acknowledgments}

The authors appreciate Assist. Prof. Kamontip Ekthamasut from the Department of Food Science and Technology, Faculty of Science, University of the Thai Chamber of Commerce, for her advice in the PCA interpretation. The authors also acknowledge the United Graduate School of Agricultural Science (UGSAS), Gifu University, Japan, for providing them some apparatus facilities. This research was financially supported by Graduate Development Scholarship 2020, National Research Council of Thailand (NRCT) (Grant no. 04/2563). The authors appreciate the Postharvest Technology Innovation Center, Ministry of Higher Education, Science, Research, and Innovation, Bangkok, for providing them some scientific facilities.

\section{Supplementary Materials}

Supplementary Figure 1: naturally ripe fruit at the initial dehiscence (red circle) at the blossom end. (Supplementary Materials) 


\section{References}

[1] C. Nualsri, K. Nakkanong, A. Chantanaorrapint, R. Rakkhan, and S. Chanaweerawan, "Genetic Diversity Analysis and Selection of Indigenous Durian in Southern Thailand," Completed Report, Faculty of Natural Resources, Print of Songkla University, Songkhla, Thailand, 2015.

[2] M. J. Brown, "Durio-a bibliographic review," in IPGRI office for South Asia, R. K. Arora, V. R. Rao, and A. N. Rao, Eds., New Delhi, India, 1997.

[3] R. Kongkachuichai, R. Charoensiri, and P. Sungpuag, "Carotenoid, flavonoid profiles and dietary fiber contents of fruits commonly consumed in Thailand," International Journal of Food Sciences \& Nutrition, vol. 61, no. 5, pp. 536-548, 2010.

[4] N. A. Husin, S. Rahman, S. Rahman, R. Karunakaran, and S. J. Bhore, "A review on the nutritional, medicinal, molecular and genome attributes of durian (Durio zibethinus L.), the king of fruits in Malaysia," Bioinformation, vol. 14, no. 6, pp. 265-270, 2018.

[5] S. Somsri, "Current status of durian breeding program in Thailand," Acta Horticulturae, vol. 1024, no. 1024, pp. 51-59, 2014.

[6] J. S. Maninang, C. Wongs-Aree, S. Kanlayanarat, S. Sugaya, and H. Gemma, "Influence of maturity and postharvest treatment on the volatile profile and physiological properties of the durian (Durio zibethinus Murray) fruit," International Food Research Journal, vol. 18, pp. 1067-1075, 2011.

[7] J. Boonthanakorn, W. Daud, A. Aontee, and C. Wongs-Aree, "Quality preservation of fresh-cut durian cv. "Monthong" using microperforated PET/PE films," Food Packaging and Shelf Life.vol. 23, Article ID 100452, 2020.

[8] N. Laohakunjit, O. Kerdchoechuen, F. B. Matta, J. L. Silva, and W. E. Holmes, "Postharvest survey of volatile compounds in five tropical fruits using headspace-solid phase microextraction (HS-SPME)," HortScience, vol. 42, no. 2, pp. 309-314, 2007.

[9] A. Niponsak, N. Laohakunjit, and O. Kerdchoechuen, "Contribution to volatile fingerprinting and physico-chemical qualities of minimally processed durian cv. "Monthong" during storage: identification of a novel chemical ripeness marker," Food and Bioprocess Technology, vol. 8, no. 6, pp. 1229-1243, 2015.

[10] P. Choosung, W. Utto, P. Boonyaritthongchai, T. Wasusri, and C. Wongs-Aree, "Ethanol vapor releasing sachet reduces decay and improves aroma attributes in mulberry fruit," Food Packaging and Shelf Life, vol. 22, Article ID 100398, 2019.

[11] P. Schieberle, "New developments in methods for analysis of volatile compounds and their precursors," in Characterization of Food: Emerging Methods, A. G. Gaonkar, Ed., Elsevier Science, The Netherlands, 1995.

[12] A. Laura, V. Luciano, G. Josep, B. Olga, and M. Montserrat, "Chemical characterization of commercial sherry vinegar aroma by headspace solid-phase microextraction and gas chromatography-olfactometry," Journal of Agricultural and Food Chemistry, vol. 59, pp. 4062-4070, 2011.

[13] J. A. Pino and S. E. Barzola-Miranda, "Characterization of odor-active compounds in pechiche (Vitex cymosa Berteo ex Speng) fruit," Journal of Raw Materials to Processed Foods, vol. 1, pp. 33-39, 2020.

[14] V. C. Allison and S. H. Katz, "An investigation of stenches and odors for industrial purposes," Journal of Industrial and Engineering Chemistry, vol. 11, no. 4, pp. 336-338, 1919.
[15] E. L. Backman, "Experimentalla undersökningar öfverluktsinnets fysiologi," Upsala Läkareförhandlingqar, vol. 22, pp. 319-470, 1917.

[16] R. G. Berger, F. Drawert, H. Kollmannsberger, S. Nitz, and B. Schraufstetter, "Novel volatiles in pineapple fruit and their sensory properties," Journal of Agricultural and Food Chemistry, vol. 33, no. 2, pp. 232-235, 1985.

[17] J. E. Cometto-Muñiz, W. S. Cain, and M. H. Abraham, "Odor detection of single chemicals and binary mixtures," Behavioural Brain Research, vol. 156, no. 1, pp. 115-123, 2005.

[18] W. Fan and Y. Xu, "Determination of odor thresholds of volatile aroma compounds in baijiu by a forced-choice ascending concentration series method of limits," Liquor Making, vol. 38, pp. 80-84, 2011.

[19] V. Ferreira, M. Ardanuy, R. López, and J. F. Cacho, "Relationship between flavor dilution values and odor unit values in hydroalcoholic solutions: role of volatility and a practical rule for its estimation," Journal of Agricultural and Food Chemistry, vol. 46, no. 10, pp. 4341-4346, 1998.

[20] L. J. van Gemert, Odour Thresholds: Compilations of Odour Threshold Values in Air, Water and Other Media, Oliemans Punter \& Partners BV, Utrecht, The Netherlands, 2011.

[21] H. Guth, "Ojectivation of white wine aromas," Thesis TU München, 1997.

[22] V. Karl, J. Gutser, A. Dietrich, B. Maas, and A. Mosandl, "Stereoisomeric flavour compounds LXVIII. 2-, 3-, and 4alkyl-branched acids, part 2: chirospecific analysis and sensory evaluation," Chirality, vol. 6, no. 5, pp. 427-434, 1994.

[23] P. Komthong, S. Hayakawa, T. Katoh, N. Igura, and M. Shimoda, "Determination of potent odorants in apple by headspace gas dilution analysis," Lebensmittel-Wissenschaft und -Technologie- Food Science and Technology, vol. 39, no. 5, pp. 472-478, 2006.

[24] M. Rychlik, P. Schieberle, and W. Grosch, Compilation of Odor Thresholds, Odor Qualities and Retention Indices of Key Food Odorants, Garching, Germany, 1998.

[25] R. Schwarz, "Über die Ricchscharfc der Honigbiene," Zeitschrift für Vergleichende Politikwissenschaft, vol. 37, pp. 180210, 1995.

[26] Y. Nagata, "Measurement of odor threshold by triangle odor bag method," in Odor Measurement Review, pp. 118-127, Office of Odor, Noise and Vibration, Ministry of the Environment, Government of Japan, Tokyo, Japan, 2003.

[27] G. R. Takeoka, R. G. Buttery, R. A. Flath et al., "Volatile constituents of pineapple (Ananas comosus [L.] Merr.)," in In Flavor Chemistry: Trends and Develpments, R. Teranishi, R. G. Buttery, and F. Shahidi, Eds., American Chemical Society, Washington, NY, USA, pp. 223-237, 1989.

[28] G. R. Takeoka, R. A. Flath, T. R. Mon, R. Teranishi, and M. Guentert, "Volatile constituents of apricot (Prunus armeniaca)," Journal of Agricultural and Food Chemistry, vol. 38, no. 2, pp. 471-477, 1990.

[29] C. Van Thriel, M. Schäper, E. Kiesswetter et al., "From chemosensory thresholds to whole body exposures-experimental approaches evaluating chemosensory effects of chemicals," International Archives of Occupational and Environmental Health, vol. 79, no. 4, pp. 308-321, 2006.

[30] P. M. Wise, T. Miyazawa, M. Gallagher, and G. Preti, "Human odor detection of homologous carboxylic acids and their binary mixtures," Chemical Senses, vol. 32, no. 5, pp. 475-482, 2007.

[31] M. Yair, Concepts in Wine Chemistry, Board and Bench Publishing Corporation, San Francisco, CA, USA, 3rd edition, 2012. 
[32] W. Hugo, E. K. Wim, and A. Anton, "Sulfur-containing volatiles of durian fruits (Durio zibethinus Murr.)," Journal of Agricultural and Food Chemistry, vol. 44, pp. 3291-3293, 1996.

[33] X. L. Jia, S. Peter, and S. Martin, "“Characterization of the major odor-active compounds in Thai durian (Durio zibethinus L. "Monthong") by aroma extract dilution analysis and headspace gas chromatography-olfactometry," Journal of Agricultural and Food Chemistry, vol. 60, pp. 11253-11262, 2012.

[34] K. Hongku, N. Laohakunjit, and O. Kerdchoechuen, "Durian flavor extracts and its volatile characteristics," Agricultural Science Journal, vol. 42, no. 2, pp. 241-244, 2011.

[35] S. C. Tongdee, A. Suwanagul, and S. Neamprem, "Durian fruit ripening and the effect of variety, maturity stage at harvest, and atmospheric gases," Acta Horticulturae, vol. 269, no. 269, pp. 323-334, 1990.

[36] Y. Y. Voon, N. Sheikh Abdul Hamid, G. Rusul, A. Osman, and S. Y. Quek, "Volatile flavour compounds and sensory properties of minimally processed durian (Durio zibethinus cv. D24) fruit during storage at $4^{\circ} \mathrm{C}$," Postharvest Biology and Technology, vol. 46, no. 1, pp. 76-85, 2007.

[37] C. Wongs-Aree and S. Noichinda, "Postharvest quality properties of potential tropical fruits related to their unique structural characters," in In Postharvest Handling: A Systems Approach, W. J. Florkowski, R. L. Shewfelt, B. Brueckner, and S. E. Prussia, Eds., Acedamic Press, Cambridge, MA, USA, 4th edition, 2021.

[38] R. G. der Agopian, J. P. Fabi, and B. R. Cordenunsi-Lysenko, "Metabolome and proteome of ethylene-treated papayas reveal different pathways to volatile compounds biosynthesis," Food Research International, vol. 131, Article ID 108975, 2020.

[39] W. Phutdhawong, S. Kaewkong, and D. Buddhasukh, "GCMS analysis of fatty acids in Thai durian aril," Chiang Mai Journal of Science, vol. 32, no. 2, pp. 155-158, 2005.

[40] A. D. Bauchot, D. S. Mottram, A. T. Dodson, and P. John, "Effect of aminocyclopropane-1-carboxylic acid oxidase antisense gene on the formation of volatile esters in cantaloupe charentais melon (cv. Védrandais)," Journal of Agricultural and Food Chemistry, vol. 46, no. 11, pp. 4787-4792, 1998.

[41] B. G. Defilippi, A. A. Kader, and A. M. Dandekar, "Apple aroma: alcohol acyltransferase, a rate limiting step for ester biosynthesis, is regulated by ethylene," Plant Science, vol. 168, no. 5, pp. 1199-1210, 2005.

[42] M. M. Khanom and Y. Ueda, "Bioconversion of aliphatic and aromatic alcohols to their corresponding esters in melons (Cucumis melo L. cv. Prince melon and cv. Earl's favorite melon)," Postharvest Biology and Technology, vol. 50, no. 1, pp. 18-24, 2008.

[43] S. Noichinda, Y. Ueda, Y. Imahori, and K. Chachin, “Thioester production and thioalcohol specificity of alcohol acetyltransferase in strawberry fruit," Food Science and Technology Research, vol. 5, no. 1, pp. 99-103, 1999.

[44] H. Kelebek, S. Selli, H. Gubbuk, and E. Gunes, "Comparative evaluation of volatiles, phenolics, sugars, organic acids and antioxidant properties of Sel-42 and Tainung papaya varieties," Food Chemistry, vol. 173, pp. 912-919, 2015. 